\title{
A note on quasilinear parabolic equations on manifolds
}

\author{
CARlo MantegazZa And LuCA MartinazZi
}

\begin{abstract}
We prove short time existence, uniqueness and continuous dependence on the initial data of smooth solutions of quasilinear locally parabolic equations of arbitrary even order on closed manifolds.
\end{abstract}

Mathematics Subject Classification (2010): 35K59 (primary); 35K41, 35K52 (secondary).

\section{Introduction}

Let $(M, g)$ be a compact, smooth Riemannian manifold without boundary of dimension $n$ and let $d \mu$ be the canonical measure associated to the metric tensor $g$.

We consider the parabolic problem with a smooth initial datum $u_{0}: M \rightarrow \mathbb{R}$,

$$
\begin{cases}u_{t}=Q[u] & \text { in } M \times[0, T] \\ u(\cdot, 0)=u_{0} & \text { on } M\end{cases}
$$

where $Q$ is a smooth, quasilinear, locally elliptic operator of order $2 p$, defined in $M \times[0, \mathcal{T}$ ) for some $\mathcal{T}>0$ which, adopting (as in the rest of the paper) the Einstein convention of summing over repeated indices, can be expressed in local coordinates as

$$
\begin{aligned}
& Q[u](x, t) \\
& =A^{i_{1} \ldots i_{2 p}}\left(x, t, u, \nabla u, \ldots, \nabla^{2 p-1} u\right) \nabla_{i_{1} \ldots i_{2 p}}^{2 p} u(x, t)+b\left(x, t, u, \nabla u, \ldots, \nabla^{2 p-1} u\right),
\end{aligned}
$$

where $A$ is a locally elliptic smooth $(2 p, 0)$-tensor of the form

$$
A^{i_{1} j_{1} \ldots i_{p} j_{p}}=(-1)^{p-1} E_{1}^{i_{1} j_{1}} \cdots E_{p}^{i_{p} j_{p}}
$$

The authors are partially supported by the Italian project FIRB-IDEAS "Analysis and Beyond". The second author is partially supported by the Swiss National Fond Grant n. PBEZP2-129520. Received October 5, 2010; accepted April 30, 2011. 
for some $(2,0)$-tensors $E_{1}, \ldots, E_{p}$ and a function $b$ smoothly depending on their arguments.

Local ellipticity here means that for every $L>0$ there exists a positive constant $\lambda \in \mathbb{R}$ such that each tensor $E_{\ell}$ satisfies

$$
E_{\ell}^{i j}\left(x, t, u, \psi_{1}, \ldots, \psi_{2 p-1}\right) \xi_{i} \xi_{j} \geq \lambda|\xi|_{g(x)}^{2}, \quad \text { for every } \xi \in T_{x}^{*} M,
$$

when $x \in M, t \in[0, T]$ with $T<\mathcal{T}, u \in \mathbb{R}$ with $|u| \leq L, \psi_{k} \in \otimes^{k} T_{x}^{*} M$ with $\left|\psi_{k}\right|_{g(x)} \leq L$. In other words we require that condition (1.3) holds for some positive $\lambda$ whenever the arguments of $E_{\ell}^{i j}$ lie in a compact set $K$ of their natural domain of definition and assume that $\lambda$ depends only on $K$. If $\lambda>0$ can be chosen independent of $K$ (i.e. of $L$ ), then we shall say that $A$ is uniformly elliptic.

Clearly, this is not the most general notion of quasilinear parabolic problems, due to the special "product" structure of the operator, anyway it covers several important situations. For instance, our definition includes the case of standard locally parabolic equations of order two in non-divergence form. Notice that we make no growth assumptions on the tensor $A$ and the function $b$.

Interchanging covariant derivatives, integrating by parts and using interpolation inequalities (see [10] for details), the following Gårding's inequality holds for this class of operators. For every smooth $u$ and $t \in[0, \mathcal{T}$ ), we have

$$
\begin{array}{r}
-\int_{M} \psi A^{i_{1} \ldots i_{2 p}}(u) \nabla_{i_{1} \ldots i_{2 p}}^{2 p} \psi d \mu \geq \sigma\|\psi\|_{W^{p, 2}(M)}^{2}-C\|\psi\|_{L^{2}(M)}^{2} \\
\forall \psi \in C^{\infty}(M),
\end{array}
$$

where the constants $\sigma>0$ and $C>0$ depend continuously only on the $C^{p}$-norm of the tensor $A$ and on the $C^{3 p-1}$-norm of the function $u$ at time $t$ (and on the curvature tensor of $(M, g)$ and its covariant derivatives). In particular, if $u$ depends smoothly on time, $\sigma=\sigma(t)$ and $C=C(t)$ are continuous functions of time.

The aim of this note is to prove the following short time existence result.

Theorem 1.1. For every $u_{0} \in C^{\infty}(M)$ there exists a positive time $T>0$ such that problem (1.1) has a smooth solution. Moreover, the solution is unique and depends continuously on $u_{0}$ in the $C^{\infty}$-topology.

Our interest in having a handy proof of this result is related to geometric evolution problems, like for instance the Ricci flow, the mean curvature flow, the Willmore flow [7], the $Q$-curvature flow [9], the Yamabe flow [4, 11, 13], etc. In all these problems, the very first step is to have a short time existence theorem showing that for an initial geometric structure (hypersurface, metric) the flow actually starts. Usually, after some manipulations in order to eliminate the degeneracies due to the geometric invariances, one has to face a quasilinear parabolic equation with smooth coefficients and smooth initial data.

If we replace the compact manifold $M$ with a bounded domain $\Omega \subset \mathbb{R}^{n}$, short time existence for quasilinear systems of order two, with prescribed boundary conditions and initial data, was proven by Giaquinta and Modica [5] in the setting of Hölder spaces. 
A different approach to Theorem 1.1 was developed by Polden in his Ph.D. Thesis [10] (see also [6]), by means of an existence result for linear equations in parabolic Sobolev spaces and the inverse function theorem. Unfortunately, as pointed out by Sharples [12], such procedure has a gap in the convergence of the solutions of the "frozen" linear problems to a solution of the quasilinear one.

In the same paper [12] Sharples, pushing further the estimates of Polden and allowing nonsmooth coefficients, was able by means of an iteration scheme to show the existence of a short time solution of the quasilinear problem on a twodimensional manifold, when the operator is of order two and in divergence form.

Our goal here is instead to simply fill the gap in Polden's proof. We start with his linear result and we show that his linearization procedure actually works if one linearizes at a suitably chosen function and discusses in details the above mentioned convergence.

As we do not assume any condition on the operator (only its product structure) and on the dimension of the manifold, we have a complete proof of the short time existence of a smooth solution to these quasilinear locally parabolic equations of arbitrary order on compact manifolds and of its uniqueness and smooth dependence on the initial data. We refer the interested reader to the nice and detailed introduction in [12] for the different approaches to the problem.

The paper is organized as follows. In the next section we present the linearization procedure, assuming Polden's linear result (Proposition 2.2 below) and we prove Theorem 1.1 by means of Lemma 2.5 which is the core of our argument. Roughly speaking, when a candidate solution $u$ stays in some parabolic Sobolev space of order high enough, the functions $u, \nabla u, \ldots, \nabla^{2 p-1} u$ are continuous (or even more regular), hence the same holds for the tensor $A$ and the function $b$. This implies that the map $u \mapsto\left(u_{t}-Q[u]\right)$ is of class $C^{1}$ between some suitable spaces, as it closely resembles a linear map with regular coefficients. This allows the application of the inverse function theorem which, in conjunction with an approximation argument, yields the existence of a solution. The last two sections are devoted to the proof of Lemma 2.5 and to the discussion of the parabolic Sobolev embeddings on which such proof relies.

We mention that the results can be extended to quasilinear parabolic systems as the linearization procedure remains the same and Polden's linear estimates (Proposition 2.2) can be actually easily generalized, assuming a suitable definition of ellipticity. In fact one easily sees that our result applies to all quasilinear systems whose linearization is invertible in the sense of Proposition 2.3 below. For more general definition of elliptic or parabolic operators of higher-order see [2].

In the following the letter $C$ will denote a constant which can change from a line to another and even within the same formula.

ACKNOWLEDGEMENTS. We are grateful to Alessandra Lunardi for useful suggestions. We wish to thank Mariano Giaquinta for several interesting discussions. 


\section{Proof of the main theorem}

We recall Polden's result for linear parabolic equations. Let us consider the problem

$$
\left\{\begin{array}{l}
u_{t}-A^{i_{1} \ldots i_{2 p} p} \nabla_{i_{1} \ldots i_{2 p}}^{2 p} u-\sum_{k=0}^{2 p-1} R_{k}^{j_{1} \ldots j_{k}} \nabla_{j_{1} \ldots j_{k}}^{k} u=b \\
u(\cdot, 0)=u_{0},
\end{array}\right.
$$

where all the tensors $A$ and $R_{k}$ depend only on $(x, t) \in M \times[0,+\infty)$, are smooth and uniformly bounded with all their derivatives. Moreover, we assume that the tensor $A$ has the product structure (1.2), where each $E_{\ell}$ is uniformly elliptic.

The Gårding's inequality for the linear operator

$$
L(u)=A^{i_{1} \ldots i_{2 p}} \nabla_{i_{1} \ldots i_{2 p}}^{2 p} u-\sum_{k=0}^{2 p-1} R_{k}^{j_{1} \ldots j_{k}} \nabla_{j_{1} \ldots j_{k}}^{k} u
$$

reads (see again [10] for details)

$$
-\int_{M} \psi L(\psi) d \mu \geq \frac{\lambda}{2}\|\psi\|_{W^{p, 2}(M)}^{2}-C\|\psi\|_{L^{2}(M)}^{2} \quad \forall \psi \in C^{\infty}(M),
$$

where the constant $C>0$ depends only on the $C^{p}$-norm of the tensors $A$ and $R_{k}$. Clearly, by approximation this inequality holds also for every $\psi \in W^{2 p, 2}(M)$.

Definition 2.1. For any $m \in \mathbb{N}$ and $a \in \mathbb{R}^{+}$we define $P_{a}^{m}(M)$ to be the completion of $C_{c}^{\infty}(M \times[0,+\infty))$ under the parabolic norm

$$
\|f\|_{P_{a}^{m}(M)}^{2}=\sum_{j, k \in \mathbb{N} \text { and } 2 p j+k \leq 2 p m} \int_{M \times[0,+\infty)} e^{-2 a t}\left|\partial_{t}^{j} \nabla^{k} f\right|^{2} d \mu d t
$$

and analogously $P^{m}(M, T)$ as the completion of $C^{\infty}(M \times[0, T])$ under the norm

$$
\|f\|_{P^{m}(M, T)}^{2}=\sum_{j, k \in \mathbb{N} \text { and } 2 p j+k \leq 2 p m} \int_{M \times[0, T]}\left|\partial_{t}^{j} \nabla^{k} f\right|^{2} d \mu d t,
$$

for every $T \in \mathbb{R}^{+}$.

Clearly for every $T \in \mathbb{R}^{+}$there is a natural continuous embedding $P_{a}^{m}(M) \hookrightarrow$ $P^{m}(M, T)$.

We have then the following global existence result for problem (2.1), by Polden [10, Theorem 2.3.5].

Proposition 2.2. For every $m \in \mathbb{N}$ there exists $a \in \mathbb{R}^{+}$large enough such that the linear map

$$
\Phi(u)=\left(u_{0}, u_{t}-A^{i_{1} \ldots i_{2 p}} \nabla_{i_{1} \ldots i_{2 p}}^{2 p} u-\sum_{k=0}^{2 p-1} R_{k}^{j_{1} \ldots j_{k}} \nabla_{j_{1} \ldots j_{k}}^{k} u\right)=\left(u_{0}, L(u)\right),
$$

where $u_{0}=u(\cdot, 0)$, is an isomorphism of $P_{a}^{m}(M)$ onto $W^{p(2 m-1), 2}(M) \times P_{a}^{m-1}(M)$. 
In the following it will be easier (though conceptually equivalent) to use the spaces $P^{m}(M, T)$ instead of the weighted spaces $P_{a}^{m}(M)$. For this reason we translate Proposition 2.2 into the setting of $P^{m}(M, T)$ spaces.

Proposition 2.3. For every $T>0$ and $m \in \mathbb{N}$ the map $\Phi$ given by formula (2.3) is an isomorphism of $P^{m}(M, T)$ onto $W^{p(2 m-1), 2}(M) \times P^{m-1}(M, T)$.

Proof. The continuity of the second component of $\Phi$ is obvious while the continuity of the first component follows as in the Polden's proof in [10, Proposition 2.2]. Hence, the map $\Phi$ is continuous, now we show that it is an isomorphism.

Given any $b \in P^{m-1}(M, T)$ we consider an extension $\widetilde{b} \in P_{a}^{m-1}(M)$ of the function $b$ and we let $\tilde{u} \in P_{a}^{m}(M)$ be the solution of problem (2.1) for $\widetilde{b}$. Clearly, $u=\left.\tilde{u}\right|_{M \times[0, T]}$ belongs to $P^{m}(M, T)$ and satisfies $\Phi(u)=\left(u_{0}, b\right)$ in $M \times[0, T]$. Suppose that $v \in P^{m}(M, T)$ is another function such that $\Phi(v)=\left(u_{0}, b\right)$ in $M \times$ $[0, T]$, then setting $w=u-v \in P^{m}(M, T)$ we have that

$$
\left\{\begin{array}{l}
w_{t}-A^{i_{1} \ldots i_{2 p}} \nabla_{i_{1} \ldots i_{2 p}}^{2 p} w-\sum_{k=0}^{2 p-1} R_{k}^{j_{1} \ldots j_{k}} \nabla_{j_{1} \ldots j_{k}}^{k} w=w_{t}-L(w)=0 \\
w(\cdot, 0)=0 .
\end{array}\right.
$$

By the very definition of solution in $P^{m}(M, T)$ (see [10]) and Gårding’s inequality (2.2), we get

$$
\begin{aligned}
\int_{M} w^{2}(x, t) d \mu(x) & =\int_{0}^{t} \int_{M} 2 w w_{t} d \mu d s \\
& =2 \int_{0}^{t} \int_{M} w L(w) d \mu d s \\
& \leq-\frac{\lambda}{2} \int_{0}^{t} \int_{M}\left|\nabla^{p} w\right|^{2} d \mu d s+C \int_{0}^{t} \int_{M} w^{2} d \mu d s \\
& \leq C \int_{0}^{t} \int_{M} w^{2}(x, s) d \mu(x) d s,
\end{aligned}
$$

as $w(\cdot, t) \in W^{2 p, 2}(M)$ for almost every $t \in[0, T]$ and where the constant $C>0$ depends only on $T$ as the coefficients of the operator $L$ are smooth. Then, by Gronwall's lemma (in its integral version) it follows that $\int_{M} w^{2}(\cdot, t) d \mu$ is zero for every $t \in[0, T]$, as it is zero at time $t=0$. It follows that $w$ is zero on all $M \times[0, T]$, hence the two functions $u$ and $v$ must coincide.

Since the map $\Phi: P^{m}(M, T) \rightarrow W^{p(2 m-1), 2}(M) \times P^{m-1}(M, T)$ is continuous, one-to-one and onto, it is an isomorphism by the open mapping theorem.

Remark 2.4. When $u_{0}$ and $b$ are smooth the unique solution $u$ of problem (2.1) belongs to all the spaces $P^{m}(M, T)$ for every $m \in \mathbb{N}$. As by Sobolev embeddings for any $k \in \mathbb{N}$ we can find a large $m \in \mathbb{N}$ so that $P^{m}(M, T)$ continuously embeds into $C^{k}(M \times[0, T])$, we can conclude that $u$ actually belongs to $C^{\infty}(M \times[0, T])$. 
Now we are ready to prove Theorem 1.1. The tensor $A$ and the function $b$ from now on will depend on $x, t, u, \nabla u, \ldots, \nabla^{2 p-1} u$ as in the introduction. Since $M$ is compact there exists a constant $C>0$ such that the initial datum satisfies $\left|u_{0}\right|+\left|\nabla u_{0}\right|_{g}+\ldots+\left|\nabla^{2 p-1} u_{0}\right|_{g} \leq C$. Then, since we are interested in existence for short time, possibly modifying the tensor $A$ and the function $b$ outside a compact set with some "cut-off" functions, we can assume that if $|u|+|\nabla u|_{g}+\ldots+\left|\nabla^{2 p-1} u\right|_{g}+$ $t \geq 2 C$, then

$$
E_{\ell}^{i j}\left(x, t, u, \nabla u, \ldots, \nabla^{2 p-1} u\right)=g^{i j}(x), \quad \text { and } \quad b\left(x, t, u, \nabla u, \ldots, \nabla^{2 p-1} u\right)=0
$$

In particular we can assume that the tensors $E_{\ell}$ are uniformly elliptic.

For a fixed $m \in \mathbb{N}$, we consider the map defined on $P^{m}(M, T)$ given by

$$
\mathcal{F}(u)=\left(u_{0}, u_{t}-Q[u]\right)=\left(u(\cdot, 0), u_{t}-A(u) \cdot \nabla^{2 p} u-b(u)\right),
$$

where in order to simplify we used the notation

$$
A(u) \cdot \nabla^{2 p} v(x, t)=A^{i_{1} \ldots i_{2 p}}\left(x, t, u(x, t), \ldots, \nabla^{2 p-1} u(x, t)\right) \nabla_{i_{1} \ldots i_{2 p}}^{2 p} v(x, t)
$$

and

$$
b(u)(x, t)=b\left(x, t, u(x, t), \ldots, \nabla^{2 p-1} u(x, t)\right)
$$

for $u, v \in P^{m}(M, T)$.

We have seen in Proposition 2.3 that if $A(u)$ and $b(u)$ only depend on $x \in M$ and $t \in[0, T]$ (and not on $u$ and its space derivatives), then $\mathcal{F}$ is a continuous map from $P^{m}(M)$ onto $W^{p(2 m-1), 2}(M) \times P^{m-1}(M)$. This is not the case in general when $A$ and $b$ depend on $u$ and its derivatives, but it is true if $m \in \mathbb{N}$ is large enough and in this case $\mathcal{F}$ is actually $C^{1}$.

Lemma 2.5. Assume that

$$
m>\frac{\operatorname{dim} M+6 p-2}{4 p}=\frac{n+6 p-2}{4 p},
$$

and $u \in P^{m}(M, T)$. Then $\mathcal{F}(u) \in W^{p(2 m-1), 2}(M) \times P^{m-1}(M, T)$ and the map

$$
\mathcal{F}: P^{m}(M, T) \rightarrow W^{p(2 m-1), 2}(M) \times P^{m-1}(M, T)
$$

is of class $C^{1}$.

We postpone the proof of this lemma to Section 3. 
We fix $m \in \mathbb{N}$ such that the hypothesis of Lemma 2.5 holds and we set

$$
\tilde{u}_{0}(x, t)=\sum_{\ell=0}^{m-1} \frac{a_{\ell}(x) t^{\ell}}{\ell !}
$$

for some functions $a_{0}, \ldots, a_{m-1} \in C^{\infty}(M)$ to be determined later. Let $w \in$ $P^{m}(M, T)$ be the unique solution of the linear problem

$$
\left\{\begin{array}{l}
w_{t}=A\left(\tilde{u}_{0}\right) \cdot \nabla^{2 p} w+b\left(\widetilde{u}_{0}\right) \\
w(\cdot, 0)=u_{0} .
\end{array}\right.
$$

Such solution exists by Proposition 2.3 and it is smooth by Remark 2.4, as $u_{0}$ and $\widetilde{u}_{0}$ are smooth (thus also $A\left(\widetilde{u}_{0}\right)$ and $b\left(\widetilde{u}_{0}\right)$ ).

Hence, we have

$\mathcal{F}(w)=\left(u_{0}, w_{t}-Q[w]\right)=\left(u_{0},\left(A\left(\widetilde{u}_{0}\right)-A(w)\right) \cdot \nabla^{2 p} w+b\left(\widetilde{u}_{0}\right)-b(w)\right)=:\left(u_{0}, f\right)$,

where we set $f=\left(A\left(\widetilde{u}_{0}\right)-A(w)\right) \cdot \nabla^{2 p} w+b\left(\widetilde{u}_{0}\right)-b(w)$.

If we compute the differential $d \mathcal{F}_{w}$ of the map $\mathcal{F}$ at the "point" $w \in C^{\infty}(M \times$ $[0, T])$, acting on $v \in P^{m}(M, T)$, we obtain

$$
\begin{aligned}
d \mathcal{F}_{w}(v)= & \left(v_{0}, v_{t}-A^{i_{1} \ldots i_{2 p}}(w) \nabla_{i_{1} \ldots i_{2 p}}^{2 p} v-D_{w} A^{i_{1} \ldots i_{2 p}}(w) v \nabla_{i_{1} \ldots i_{2 p}}^{2 p} w \ldots\right. \\
& \cdots-D_{w_{j_{1} \ldots j_{2 p-1}}} A^{i_{1} \ldots i_{2 p}}(w) \nabla_{j_{1} \ldots j_{2 p-1}}^{2 p-1} v \nabla_{i_{1} \ldots i_{2 p}}^{2 p} w \\
& \left.-D_{w} b(w) v \cdots-D_{w_{j_{1} \ldots j_{2 p-1}}} b(w) \nabla_{j_{1} \ldots j_{2 p-1}}^{2 p-1} v\right)
\end{aligned}
$$

where $v_{0}=v(\cdot, 0)$ and we denoted by $D_{w_{j_{1} \ldots j_{k}}} A^{i_{1} \ldots i_{2 p}}(w), D_{w_{j_{1} \ldots j_{k}}} b(w)$ the derivatives of the tensor $A$ and of the function $b$ with respect to their variables $\nabla_{j_{1} \ldots j_{k}}^{k} w$, respectively.

Then, we can see that $d \mathcal{F}_{w}(v)=(z, h) \in W^{p(2 m-1), 2}(M) \times P^{m-1}(M, T)$ implies that $v$ is a solution of the linear problem

$$
\left\{\begin{array}{l}
v_{t}-\widetilde{A}^{i_{1} \ldots i_{2 p} p} \nabla_{i_{1} \ldots i_{2 p}}^{2 p} v-\sum_{k=0}^{2 p-1} \widetilde{R}_{k}^{j_{1} \ldots j_{k}} \nabla_{j_{1} \ldots j_{k}}^{k} v=h \\
v(\cdot, 0)=z,
\end{array}\right.
$$

where

$$
\begin{aligned}
\widetilde{A}^{i_{1} \ldots i_{2 p}} & =A^{i_{1} \ldots i_{2 p}}(w), \\
\widetilde{R}_{k}^{j_{1} \ldots j_{k}} & =D_{w_{j_{1} \ldots j_{k}}} A^{i_{1} \ldots i_{2 p}}(w) \nabla_{i_{1} \ldots i_{2 p}}^{2 p} w+D_{w_{j_{1} \ldots j_{k}}} b(w)
\end{aligned}
$$

are smooth tensors independent of $v$. 
By Proposition 2.3 for every $(z, h) \in W^{p(2 m-1), 2}(M) \times P^{m-1}(M, T)$ there exists a unique solution $v$ of this problem, hence $d \mathcal{F}_{w}$ is a Hilbert space isomorphism and the inverse function theorem can be applied, as the map $\mathcal{F}$ is $C^{1}$ by Lemma 2.5. Hence, the map $\mathcal{F}$ is a diffeomorphism of a neighborhood $U \subset P^{m}(M, T)$ of $w$ onto a neighborhood $V \subset W^{p(2 m-1), 2}(M) \times P^{m-1}(M, T)$ of $\left(u_{0}, f\right)$.

Getting back to the functions $a_{\ell}$, we claim that we can choose them such that $a_{\ell}=\left.\partial_{t}^{\ell} w\right|_{t=0} \in C^{\infty}(M)$ for every $\ell=0, \ldots, m-1$.

We apply the following recurrence procedure. We set $a_{0}=u_{0} \in C^{\infty}(M)$ and, assuming to have defined $a_{0}, \ldots, a_{\ell}$, we consider the derivative

$$
\begin{aligned}
\left.\partial_{t}^{\ell+1} w\right|_{t=0}=\partial_{t}^{\ell}[ & A^{i_{1} \ldots i_{2 p}}\left(x, t, \tilde{u}_{0}, \nabla \tilde{u}_{0}, \ldots, \nabla^{2 p-1} \tilde{u}_{0}\right) \nabla_{i_{1} \ldots i_{2 p}}^{2 p} w \\
& \left.+b\left(x, t, \tilde{u}_{0}, \nabla \tilde{u}_{0}, \ldots, \nabla^{2 p-1} \widetilde{u}_{0}\right)\right]\left.\right|_{t=0}
\end{aligned}
$$

and we see that the right-hand side contains time-derivatives at time $t=0$ of $\tilde{u}_{0}, \ldots, \nabla^{2 p-1} \tilde{u}_{0}$ and $\nabla_{i_{1} \ldots i_{2 p}}^{2 p} w$ only up to the order $\ell$, hence it only depends on the functions $a_{0}, \ldots, a_{\ell}$. Then, we define $a_{\ell+1}$ to be equal to such expression. Iterating up to $m-1$, the set of functions $a_{0}, \ldots, a_{m-1}$ satisfies the claim.

Then, $a_{\ell}=\left.\partial_{t}^{\ell} \widetilde{u}_{0}\right|_{t=0}=\left.\partial_{t}^{\ell} w\right|_{t=0}$ and it easily follows by the "structure" of the function $f \in C^{\infty}(M \times[0, T])$, that we have $\left.\partial_{t}^{\ell} f\right|_{t=0}=0$ and $\left.\nabla^{j} \partial_{t}^{\ell} f\right|_{t=0}=0$ for any $0 \leq \ell \leq m-1$ and $j \in \mathbb{N}$.

We consider now for any $k \in \mathbb{N}$ the "translated" functions $f_{k}: M \times[0, T] \rightarrow \mathbb{R}$ given by

$$
f_{k}(x, t)= \begin{cases}0 & \text { if } t<1 / k \\ f(x, t-1 / k) & \text { if } 1 / k \leq t \leq T .\end{cases}
$$

Since $f \in C^{\infty}(M \times[0, T])$ and $\left.\nabla^{j} \partial_{t}^{\ell} f\right|_{t=0}=0$ for every $0 \leq \ell \leq m-1$ and every $j \in \mathbb{N}$, all the functions $\nabla^{j} \partial_{t}^{\ell} f_{k} \in C^{0}(M \times[0, T])$ for every $0 \leq \ell \leq m-1$ and $j \geq 0$, it follows easily that

$$
\nabla^{j} \partial_{t}^{\ell} f_{k} \rightarrow \nabla^{j} \partial_{t}^{\ell} f \quad \text { in } L^{2}(M \times[0, T]) \text { for } 0 \leq \ell \leq m-1, j \geq 0,
$$

hence $f_{k} \rightarrow f$ in $P^{m}(M, T)$.

Hence, there exists a function $\tilde{f} \in P^{m-1}(M, T)$ such that $\left(u_{0}, \tilde{f}\right)$ belongs to the neighborhood $V$ of $\mathcal{F}(w)$ and $\widetilde{f}=0$ in $M \times\left[0, T^{\prime}\right]$ for some $T^{\prime} \in(0, T]$. Since $\left.\mathcal{F}\right|_{U}$ is a diffeomorphism between $U$ and $V$, we can find a function $u \in U$ such that $\mathcal{F}(u)=\left(u_{0}, \widetilde{f}\right)$. Clearly such $u \in P^{m}\left(M, T^{\prime}\right)$ is a solution of problem (1.1) in $M \times\left[0, T^{\prime}\right]$. Since $u \in P^{m}\left(M, T^{\prime}\right)$ implies that $\nabla^{2 p-1} u \in C^{0}\left(M \times\left[0, T^{\prime}\right]\right)$, parabolic regularity implies that actually $u \in C^{\infty}\left(M \times\left[0, T^{\prime}\right]\right)$.

We now prove uniqueness by a standard energy estimate, which we include for completeness. In the sequel for simplicity we relabel $T$ the time $T^{\prime}$ found above. 
Suppose that we have two smooth solutions $u, v: M \times[0, T] \rightarrow \mathbb{R}$ of Problem (1.1). Setting $w:=u-v$, we compute in an orthonormal frame

$$
\begin{aligned}
\frac{d}{d t} \int_{M}\left|\nabla^{p} w\right|^{2} d \mu= & \int_{M} 2 \nabla_{i_{1} \ldots i_{p}}^{p} w \nabla_{i_{1} \ldots i_{p}}^{p}\left(A(u) \cdot \nabla^{2 p} u-A(v) \cdot \nabla^{2 p} v\right) d \mu \\
& +\int_{M} 2 \nabla_{i_{1} \ldots i_{p}}^{p} w \nabla_{i_{1} \ldots i_{p}}^{p}(b(u)-b(v)) d \mu \\
= & 2 \int_{M} \nabla_{i_{1} \ldots i_{p}}^{p} w \nabla_{i_{1} \ldots i_{p}}^{p}\left(A(u) \cdot \nabla^{2 p} w\right) d \mu \\
& +2 \int_{M} \nabla_{i_{1} \ldots i_{p}}^{p} w \nabla_{i_{1} \ldots i_{p}}^{p}\left((A(u)-A(v)) \cdot \nabla^{2 p} v\right) d \mu \\
& +2(-1)^{p} \int_{M} \nabla_{i_{1} \ldots i_{p}}^{p} \nabla_{i_{1} \ldots i_{p}}^{p} w(b(u)-b(v)) d \mu \\
\leq & 2 \int_{M} \nabla_{i_{1} \ldots i_{p}}^{p} w \nabla_{i_{1} \ldots i_{p}}^{p}\left(A(u) \cdot \nabla^{2 p} w\right) d \mu \\
& +2 \int_{M}\left|\nabla^{2 p} w\right|\left(|A(u)-A(v)|\left|\nabla^{2 p} v\right|+|b(u)-b(v)|\right) d \mu,
\end{aligned}
$$

where the integrals over $M$ are intended at time $t \in[0, T]$.

Now we consider the integral $\int_{M} \nabla_{i_{1} \ldots i_{p}}^{p} w \nabla_{i_{1} \ldots i_{p}}^{p}\left(A^{j_{1} \ldots j_{2 p}}(u) \nabla_{j_{1} \ldots j_{2} p}^{2 p} w\right) d \mu$. Expanding the derivative $\nabla_{i_{1} \ldots i_{p}}^{p}\left(A^{j_{1} \ldots j_{2} p}(u) \nabla_{j_{1} \ldots j_{2} p}^{2 p} w\right)$ we will get one special term $A^{j_{1} \ldots j_{2} p} \quad(u) \nabla_{i_{1} \ldots i_{p} j_{1} \ldots j_{2}}^{3 p} w$ and several other terms of the form $B\left(x, t, u, \ldots, \nabla^{3 p-1} u\right) \# \nabla^{q} w$ with $2 p \leq q<3 p$, for some tensor $B$ smoothly depending on its arguments, where the symbol \# means metric contraction on some indices. For each of these terms, integrating repeatedly by parts, we can write

$$
\begin{aligned}
& \int_{M} \nabla^{p} w \# B\left(x, t, u, \ldots, \nabla^{3 p-1} u\right) \# \nabla^{q} w d \mu \\
& =\sum_{\ell=p}^{2 p} \int_{M} \nabla^{\ell} w \# D_{\ell}\left(x, t, u, \ldots, \nabla^{4 p-1} u\right) \# \nabla^{q-p} w d \mu
\end{aligned}
$$

where the tensors $D_{\ell}$ are smoothly depending on their arguments.

Since $u \in C^{\infty}(M \times[0, T])$, all the tensors $D_{\ell}$ are bounded, hence we can estimate

$$
\begin{aligned}
\int_{M} \nabla_{i_{1} \ldots i_{p}}^{p} w \nabla_{i_{1} \ldots i_{p}}^{p}\left(A(u) \cdot \nabla^{2 p} w\right) d \mu \leq & \int_{M} \nabla_{i_{1} \ldots i_{p}}^{p} w A^{j_{1} \ldots j_{2 p}}(u) \nabla_{i_{1} \ldots i_{p} j_{1} \ldots j_{2 p}}^{3 p} w d \mu \\
& +C \sum_{r=p}^{2 p-1} \sum_{\ell=p}^{2 p} \int_{M}\left|\nabla^{\ell} w\right|\left|\nabla^{r} w\right| d \mu .
\end{aligned}
$$


where $C$ is a constant independent of time (actually $C$ depends only on the structure of $A$ ). Interchanging the covariant derivatives we have

$$
\nabla_{i_{1} \ldots i_{p} j_{1} \ldots j_{2} p}^{3 p} w=\nabla_{j_{1} \ldots i_{2 p} i_{1} \ldots i_{p}}^{3 p} w+\sum_{q=0}^{3 p-1} R_{q} \# \nabla^{q} w
$$

where the tensors $R_{q}$ are functions of the Riemann tensor and its covariant derivatives, hence they are smooth and bounded. We can clearly deal with this sum of terms as above, by means of integrations by parts, obtaining the same result. Then we conclude, also using Gårding's inequality (1.4)

$$
\begin{aligned}
& \int_{M} \nabla_{i_{1} \ldots i_{p}}^{p} w \nabla_{i_{1} \ldots i_{p}}^{p}\left(A(u) \cdot \nabla^{2 p} w\right) d \mu \leq \int_{M} \nabla_{i_{1} \ldots i_{p}}^{p} w A^{j_{1} \ldots j_{2} p}(u) \nabla_{j_{1} \ldots j_{2} p}^{2 p} \nabla_{i_{1} \ldots i_{p}}^{p} w d \mu \\
& +C \sum_{r=p}^{2 p-1} \sum_{\ell=p}^{2 p} \int_{M}\left|\nabla^{\ell} w\right|\left|\nabla^{r} w\right| d \mu \\
& \leq-\alpha \int_{M}\left|\nabla^{2 p} w\right|^{2} d \mu+C \sum_{r=p}^{2 p-1} \sum_{\ell=p}^{2 p} \int_{M}\left|\nabla^{\ell} w\right|\left|\nabla^{r} w\right| d \mu,
\end{aligned}
$$

for some positive constant $\alpha$. Getting back to the initial computation and using Peter-Paul inequality we get

$$
\begin{aligned}
\frac{d}{d t} \int_{M}\left|\nabla^{p} w\right|^{2} d \mu \leq & -2 \alpha \int_{M}\left|\nabla^{2 p} w\right|^{2} d \mu+C \sum_{r=p}^{2 p-1} \sum_{\ell=p}^{2 p} \int_{M}\left|\nabla^{\ell} w\right|\left|\nabla^{r} w\right| d \mu \\
& +C \int_{M}\left|\nabla^{2 p} w\right|\left(|A(u)-A(v)|\left|\nabla^{2 p} v\right|+|b(u)-b(v)|\right) d \mu \\
\leq & -2 \alpha \int_{M}\left|\nabla^{2 p} w\right|^{2} d \mu+C \sum_{r=p}^{2 p-1} \sum_{\ell=p}^{2 p-1} \int_{M}\left|\nabla^{\ell} w\right|\left|\nabla^{r} w\right| d \mu \\
& +\sum_{r=0}^{2 p-1}\left(\varepsilon_{r} \int_{M}\left|\nabla^{2 p} w\right|^{2} d \mu+C_{\varepsilon_{r}} \int_{M}\left|\nabla^{r} w\right|^{2} d \mu\right) \\
& +\delta \int_{M}\left|\nabla^{2 p} w\right|^{2} d \mu+C_{\delta} \int_{M}\left(|A(u)-A(v)|^{2}+|b(u)-b(v)|^{2}\right) d \mu \\
\leq & -\alpha \int_{M}\left|\nabla^{2 p} w\right|^{2} d \mu+C \sum_{r=0}^{2 p-1} \int_{M}\left|\nabla^{r} w\right|^{2} d \mu \\
& +C_{\delta} \int_{M}\left(|A(u)-A(v)|^{2}+|b(u)-b(v)|^{2}\right) d \mu,
\end{aligned}
$$

where we chose $\delta+\sum_{r=0}^{2 p-1} \varepsilon_{r}=\alpha$ and we used the fact that $\left|\nabla^{2 p} v\right|$ is bounded. 
As the tensor $A$ and the function $b$ are smooth, we can easily bound

$$
|A(u)-A(v)|^{2}+\left|b(u)-b(v)^{2}\right| \leq C \sum_{r=0}^{2 p-1}\left|\nabla^{r} u-\nabla^{r} v\right|^{2}=C \sum_{r=0}^{2 p-1}\left|\nabla^{r} w\right|^{2}
$$

so finally

$$
\frac{d}{d t} \int_{M}\left|\nabla^{p} w\right|^{2} d \mu \leq-\alpha \int_{M}\left|\nabla^{2 p} w\right|^{2} d \mu+C \sum_{r=0}^{2 p-1} \int_{M}\left|\nabla^{r} w\right|^{2} d \mu .
$$

Now we have, using again Gårding's and Peter-Paul inequalities,

$$
\begin{aligned}
& \left.\frac{d}{d t} \int_{M} w^{2} d \mu=2 \int_{M} w\left(A(u) \cdot \nabla^{2 p} u-A(v) \cdot \nabla^{2 p} v\right) d \mu+2 \int_{M} w(b(u)-b(v))\right) d \mu \\
& =2 \int_{M} w A(u) \cdot \nabla^{2 p} w d \mu+2 \int_{M} w\left((A(u)-A(v)) \cdot \nabla^{2 p} v+b(u)-b(v)\right) d \mu \\
& \leq-\beta \int_{M}\left|\nabla^{p} w\right|^{2} d \mu+C \int_{M} w^{2} d \mu+C \int_{M} w(A(u)-A(v)+b(u)-b(v)) d \mu \\
& \leq-\beta \int_{M}\left|\nabla^{p} w\right|^{2} d \mu+C \int_{M} w^{2} d \mu+C \int_{M}\left(|A(u)-A(v)|^{2}+|b(u)-b(v)|^{2}\right) d \mu .
\end{aligned}
$$

Estimating the last integral as before and putting the two computation together we obtain

$$
\frac{d}{d t} \int_{M}\left(\left|\nabla^{p} w\right|^{2}+w^{2}\right) d \mu \leq-\frac{\alpha}{2} \int_{M}\left|\nabla^{2 p} w\right|^{2} d \mu+C \sum_{r=0}^{2 p-1} \int_{M}\left|\nabla^{r} w\right|^{2} d \mu .
$$

In order to deal with the last term, we apply the following Gagliardo-Nirenberg interpolation inequalities (see [3, Proposition 2.11] and [1, Theorem 4.14]): for every $0 \leq r<2 p$ and $\varepsilon>0$ there exists a constant $C_{\varepsilon}$ such that

$$
\left\|\nabla^{r} f\right\|_{L^{2}(M)}^{2} \leq \varepsilon\left\|\nabla^{2 p} f\right\|_{L^{2}(M)}^{2}+C_{\varepsilon}\|f\|_{L^{2}(M)}^{2}
$$

for every function $f \in W^{2 p, 2}(M)$.

Hence, for some $\varepsilon>0$ small enough we get,

$$
\begin{aligned}
& \frac{d}{d t} \int_{M}\left(\left|\nabla^{p} w\right|^{2}+w^{2}\right) d \mu \\
& \leq-\frac{\alpha}{4} \int_{M}\left|\nabla^{2 p} w\right|^{2} d \mu+C \sum_{r=0}^{2 p-1} \varepsilon \int_{M}\left|\nabla^{2 p} w\right|^{2} d \mu+C \sum_{r=0}^{2 p-1} C_{\varepsilon} \int_{M} w^{2} d \mu \\
& \leq C \int_{M} w^{2} d \mu .
\end{aligned}
$$


From this ordinary differential inequality and Gronwall's lemma, it follows that if the quantity $\int_{M}\left(\left|\nabla^{p} w\right|^{2}+w^{2}\right) d \mu$ is zero at some time $t_{0}$, then it must be zero for every time $t \in\left[t_{0}, T\right]$. Since at $t=0$ we have $w(\cdot, 0)=u_{0}-v_{0}=0$, we are done.

We now prove the continuous dependence of a solution $u \in C^{\infty}(M \times[0, T])$ on its initial datum $u_{0}=u(\cdot, 0) \in C^{\infty}(M)$. Fix any $m \in \mathbb{N}$ satisfying condition (2.4), so that by the Sobolev embeddings $u \in P^{m}(M, T)$ implies $\nabla^{2 p-1} u \in C^{0}(M \times$ $[0, T])$. By the above argument, $u=\left(\left.\mathcal{F}\right|_{U}\right)^{-1}\left(u_{0}, 0\right) \in P^{m}(M, T)$ where $\left.\mathcal{F}\right|_{U}$ is a diffeomorphism of an open set $U \subset P^{m}(M, T)$ onto $V \subset W^{p(2 m-1), 2}(M) \times$ $P^{m-1}(M, T)$, with $\left(u_{0}, 0\right) \in V$. Then, assuming that $u_{k, 0} \rightarrow u_{0}$ in $C^{\infty}(M)$ as $k \rightarrow \infty$, we also have $u_{k, 0} \rightarrow u_{0}$ in $W^{p(2 m-1), 2}(M)$, hence for $k$ large enough $\left(u_{k, 0}, 0\right) \in V$ and there exists $u_{k} \in U$ such that $\mathcal{F}\left(u_{k}\right)=\left(u_{k, 0}, 0\right)$. This is the unique solution in $P^{m}(M, T)$ (hence in $C^{\infty}(M \times[0, T])$ by parabolic bootstrap) with initial datum $u_{k, 0}$. Moreover, since $\left.\mathcal{F}\right|_{U}$ is a diffeomorphism, we have $u_{k} \rightarrow u$ in $P^{m}(M, T)$.

By uniqueness, we can repeat the same procedure for any $m \in \mathbb{N}$ satisfying condition (2.4) concluding that $u_{k} \rightarrow u$ in $P^{m}(M, T)$ for every such $m \in \mathbb{N}$, hence in $C^{\infty}(M \times[0, T])$.

\section{Proof of Lemma 2.5}

We shall write $P^{m}=P^{m}(M, T), L^{q}=L^{q}(M \times[0, T]), C^{0}=C^{0}(M \times[0, T])$ etc..., so that for instance $C^{0}\left(P^{m} ; C^{1}\right)$ will denote the space of continuous maps from $P^{m}(M, T)$ to $C^{1}(M \times[0, T])$. The first component of $\mathcal{F}$, i.e. the map $u \mapsto u(\cdot, 0)$ is linear and bounded from $P^{m}$ to $W^{p(2 m-1), 2}(M)$, by Proposition 2.3, therefore it is $C^{1}$. Obviously the map $u \mapsto \partial_{t} u$ is linear and bounded from $P^{m}$ to $P^{m-1}$, hence also $C^{1}$. Thus, it remains to show that the two maps

$$
\mathcal{F}_{A}(u):=A(u) \cdot \nabla^{2 p} u, \quad \mathcal{F}_{b}(u):=b(u)
$$

belong to $C^{1}\left(P^{m} ; P^{m-1}\right)$.

We first prove that $\mathcal{F}_{A}, \mathcal{F}_{b} \in C^{0}\left(P^{m} ; P^{m-1}\right)$. By an induction argument, it is easy to see that for every $k \in \mathbb{N}$

$$
\nabla^{k}\left(A(u) \cdot \nabla^{2 p} u\right)=\sum_{j=0}^{k} \sum_{\substack{i_{1}, i_{2}, \ldots, i_{j+1} \geq 1 \\ i_{1}+\cdots+i_{j+1} \leq k+2 p+(2 p-1) j}} \partial^{j} A(u) \# \nabla^{i_{1}} u \# \ldots \# \nabla^{i_{j+1}} u,
$$

where $\partial^{j} A(u)$ denotes the $j$-th derivative of $A$ with respect to any of its arguments and $D \# E$ denotes an arbitrary contraction with the metric of two tensors $D$ and $E$.

Taking into account formula (3.1) with $k \leq 2 p(m-1)$, in order to prove that the map $u \mapsto \nabla^{2 p(m-1)}\left(A(u) \cdot \nabla^{2 p} u\right)$ belongs to $C^{0}\left(P^{m} ; L^{2}\right)$ we have to show that any map of the form

$$
u \mapsto \partial^{j} A(u) \# \nabla^{i_{1}} u \# \cdots \# \nabla^{i_{j+1}} u
$$


belongs to $C^{0}\left(P^{m} ; L^{2}\right)$ whenever

$$
i_{1}+\cdots+i_{j+1} \leq 2 p m+(2 p-1) j \quad \text { and } \quad i_{1}, \ldots, i_{j+1} \geq 1 .
$$

The case $r=0$ and $\ell=2 p-1$ of the Sobolev embeddings (4.3) below and condition (2.4) imply that if $u \in P^{m}$ then $\nabla^{2 p-1} u \in C^{0}$ (and the immersion is bounded), hence all the maps $u \mapsto \partial^{j} A(u)$ belong to $C^{0}\left(P^{m} ; C^{0}\right)$.

We can assume from now on that $j \geq 1$, since in the case $j=0$, we get the term $A(u) \# \nabla^{2 p+k} u$ which is continuous from $P^{m}$ to $L^{2}$ as a function of $u$ for $k \leq 2 p(m-1)$.

As for the factors $\nabla^{i_{\ell}} u$ appearing in formula (3.2), first we assume that each $i_{\ell}$ is such that we are in case (4.1) of Sobolev embeddings, i.e.

$$
\frac{1}{q_{\ell}}:=\frac{1}{2}-\frac{2 p m-i_{\ell}}{n+2 p}>0,
$$

so that the map $u \mapsto \nabla^{i_{\ell}} u$ lies in $C^{0}\left(P^{m} ; L^{q_{\ell}}\right)$. By Hölder's inequality, the condition

$$
\frac{1}{q}:=\sum_{\ell=1}^{j+1} \frac{1}{q_{\ell}}=\sum_{\ell=1}^{j+1}\left(\frac{1}{2}-\frac{2 p m-i_{\ell}}{n+2 p}\right) \leq \frac{1}{2},
$$

implies that the map $u \mapsto \nabla^{i_{1}} u \# \ldots \# \nabla^{i_{j+1}} u$ belongs to $C^{0}\left(P^{m} ; L^{q}\right)$, hence also to $C^{0}\left(P^{m} ; L^{2}\right)$, as $L^{q}$ embeds continuously into $L^{2}$ for $q \geq 2$. Then, if we show inequality (3.5), the map defined by formula (3.2) belongs to $C^{0}\left(P^{m} ; L^{2}\right)$. From inequalities (2.4), (3.3) and $j \geq 1$ it follows,

$$
\begin{aligned}
\sum_{\ell=1}^{j+1} \frac{1}{q_{\ell}} & \leq \frac{j+1}{2}-\frac{2 p m(j+1)-2 p m-(2 p-1) j}{n+2 p} \\
& =\frac{1}{2}+\frac{j}{2}-\frac{(2 p m-2 p+1) j}{n+2 p}<\frac{1}{2} .
\end{aligned}
$$

Now, if for some $i_{\ell}$, say $i_{1}, \ldots, i_{s}$, we have $\frac{2 p m-i_{\ell}}{n+2 p}>\frac{1}{2}$, then we are in case (4.3) of Sobolev embeddings and the corresponding maps $u \mapsto \nabla^{i_{\ell}} u$ belong to $C^{0}\left(P^{m} ; C^{0}\right)$, hence we can avoid to estimate such factors, as for $A(u)$. Then, since (3.4) holds for $\ell \in\{s+1, \ldots, j+1\}$, arguing again by induction, in this case we have to deal with functions $u \mapsto \nabla^{i_{s+1}} u \# \cdots \# \nabla^{i_{j+1}} u$ under the conditions

$$
i_{s+1}+\cdots+i_{j+1} \leq 2 p m+(2 p-1)(j-s) \quad \text { and } \quad i_{s+1}, \ldots i_{j+1} \geq 1 .
$$

Then, computing as in inequality (3.6) one shows

$$
\begin{aligned}
\sum_{\ell=s+1}^{j+1} \frac{1}{q_{\ell}} & \leq \frac{j+1-s}{2}-\frac{2 p m(j+1-s)-2 p m-(2 p-1)(j-s)}{n+2 p} \\
& =\frac{1}{2}+\frac{j-s}{2}-\frac{(2 p m-2 p+1)(j-s)}{n+2 p} \\
& \leq \frac{1}{2}
\end{aligned}
$$


where we intend that if $s=j+1$ there is nothing to sum. Notice that the last inequality is strict if $s \neq j$, and in the case $s=j$ the map $u \mapsto \nabla^{i j+1} u$ is continuous from $P^{m}$ to $L^{2}$ as $i_{j+1} \leq 2 p m$.

If in addition for some $i_{\ell}$, say $i_{s+1}, \ldots, i_{r}$, we have $\frac{2 p m-i_{\ell}}{n+2 p}=\frac{1}{2}$ (i.e. we are in the critical case (4.2) of the Sobolev embeddings), we know that for such indices the maps $u \mapsto \nabla^{i \ell} u$ belong to $C^{0}\left(P^{m} ; L^{q}\right)$ for every $1 \leq q<\infty$. Then inequality (3.7) still holds true if we choose $q_{s+1}, \ldots, q_{r}$ large enough, since, unless $s=r=j$, the last inequality in (3.7) is strict.

Hence, we conclude as before that the map $u \mapsto \nabla^{2 p(m-1)}\left(A(u) \cdot \nabla^{2 p} u\right)$ lies in $C^{0}\left(P^{m} ; L^{2}\right)$.

The time or mixed space-time derivatives $\partial_{t}^{r} \nabla^{k}\left(A(u) \cdot \nabla^{2 p} u\right)$ with $2 p r+k \leq$ $2 p(m-1)$ can be treated in a similar way, observing that the functions $\partial_{t}^{r} \nabla^{\ell} u$ have the same integrability of $\nabla^{2 p r+\ell} u$ from the point of view of the embeddings (4.1)(4.3).

Starting from formula (3.1) and differentiating in time, again by an induction argument, one gets

$$
\begin{aligned}
& \partial_{t}^{r} \nabla^{k}\left(A(u) \cdot \nabla^{2 p} u\right) \\
& =\sum_{j=0}^{r+k} \sum_{\begin{array}{c}
i_{1}, \ldots, i_{j+1}, \iota_{1}, \ldots, l_{j+1} \geq 0 \\
i_{1}+\cdots+i_{j+1} \leq k+2 p+(2 p-1) j \\
l_{1}+\cdots+\iota_{j+1} \leq r
\end{array}} \partial^{j} A(u) \# \partial_{t}^{l_{1}} \nabla^{i_{1}} u \# \cdots \# \partial_{t}^{l_{j+1}} \nabla^{i_{j+1}} u . \\
&
\end{aligned}
$$

Then, with the same proof as before one shows that a map of the form

$$
u \mapsto \partial^{j} A(u) \# \partial_{t}^{\iota_{1}} \nabla^{i_{1}} u \# \cdots \# \partial_{t}^{\iota_{j+1}} \nabla^{i_{j+1}} u
$$

belongs to $C^{0}\left(P^{m+1} ; L^{2}\right)$ whenever $i_{1}, \ldots, i_{j+1}, \iota_{1}, \ldots, \iota_{j+1} \geq 0$ and

$$
i_{1}+\cdots+i_{j+1}+2 p\left(\iota_{1}+\cdots+\iota_{j+1}\right) \leq 2 p m+(2 p-1) j .
$$

Hence the map $u \mapsto \partial_{t}^{r} \nabla^{k}\left(A(u) \cdot \nabla^{2 p} u\right)$ belongs to $C^{0}\left(P^{m} ; L^{2}\right)$ for $2 p r+k \leq$ $2 p(m-1)$, which means that $\mathcal{F}_{A} \in C^{0}\left(P^{m} ; P^{m-1}\right)$ as wished.

The map $\mathcal{F}_{b}$ can be treated in a similar way, so also $\mathcal{F}_{b} \in C^{0}\left(P^{m} ; P^{m-1}\right)$. It remains to prove that $d \mathcal{F}_{A}, d \mathcal{F}_{b} \in C^{0}\left(P^{m} ; L\left(P^{m} ; P^{m-1}\right)\right)$, where $L\left(P^{m} ; P^{m-1}\right)$ denotes the Banach space of bounded linear maps from $P^{m}$ into $P^{m-1}$. We first claim that the Gateaux derivative

$$
(u, v) \mapsto d \mathcal{F}_{A}(u)(v):=\left.\frac{d}{d t} \mathcal{F}_{A}(u+t v)\right|_{t=0}
$$

belongs to $C^{0}\left(P^{m} \times P^{m} ; P^{m-1}\right)$. Indeed, $d \mathcal{F}_{A}(u)(v)$ can be written as

$$
B(u, v) \# \nabla^{2 p} u+A(u) \cdot \nabla^{2 p} v,
$$


where $B$ is a tensor depending smoothly on $x, t, u, \ldots, \nabla^{2 p-1} u$ and linearly on some derivative of $v$ up to the order $2 p-1$, that is, $B(u, v)=\sum_{\ell=0}^{2 p-1} B_{\ell}(u) \cdot \nabla^{\ell} v$, compare with formula (2.5). The estimates proven for $\mathcal{F}_{A}$ can be applied to any term of the form $\partial_{t}^{r} \nabla^{k}\left(B(u, v) \# \nabla^{2 p} u\right)$, since they can be expressed as a sum similar to the right-hand side of identity (3.8). The only difference is that now in every term of such sum one linear occurrence of $u$ is replaced by $v$. Precisely, writing $u_{1}:=u$, $u_{2}:=v$ every term $\partial^{j} A(u) \# \partial_{t}^{l_{1}} \nabla^{i_{1}} u \# \cdots \# \partial_{t}^{l_{j+1}} \nabla^{i_{j+1}} u$ has to be replaced by some

$$
D(u) \# \partial_{t}^{l_{1}} \nabla^{i_{1}} u_{\tau_{1}} \# \cdots \# \partial_{t}^{l_{j+1}} \nabla^{i_{j+1}} u_{\tau_{j+1}}
$$

where exactly one of the indices $\tau_{1}, \ldots, \tau_{j+1}$ is equal to 2 , and the others are equal to 1 .

An analogous reasoning applies to the term $A(u) \cdot \nabla^{2 p} v$. It is then easy to see, since $v \in P^{m}$ like $u$, that we can repeat the same estimates used to show the continuity of $u \mapsto \mathcal{F}_{A}(u)$. This proves in particular that $d \mathcal{F}_{A}(u) \in L\left(P^{m} ; P^{m-1}\right)$.

In order now to prove that $d \mathcal{F}_{A} \in C^{0}\left(P^{m} ; L\left(P^{m} ; P^{m-1}\right)\right)$ we need to show that

$$
\sup _{\|v\|_{P m} \leq 1}\left\|d \mathcal{F}_{A}(\widetilde{u})(v)-d \mathcal{F}_{A}(u)(v)\right\|_{P^{m-1}} \rightarrow 0 \quad \text { as } \tilde{u} \rightarrow u \text { in } P^{m} .
$$

Again, this estimate is similar to what we have already done. Indeed, supposing that $\tau_{j+1}$ is the only index equal to 2 in (3.11) and assuming that there are no time derivatives for the sake of simplicity, we want to see that, as $\widetilde{u} \rightarrow u$ in $P^{m}$,

$\sup \left\|D(\widetilde{u}) \# \nabla^{i_{1}} \widetilde{u} \# \cdots \# \nabla^{i_{j}} \widetilde{u} \nabla^{i_{j+1}} v-D(u) \# \nabla^{i_{1}} u \# \cdots \# \nabla^{i_{j}} u \nabla^{i_{j+1}} v\right\|_{L^{2}} \rightarrow 0$, (3.12) $\|v\|_{P} \leq 1$

where $i_{1}+\cdots+i_{j+1} \leq 2 p m+(2 p-1) j$ (see formula (3.1) and condition (3.3)).

Adding and subtracting terms, one gets

$$
\begin{aligned}
& \left|D(\widetilde{u}) \# \nabla^{i_{1}} \widetilde{u} \# \cdots \# \nabla^{i_{j}} \widetilde{u} \nabla^{i_{j+1}} v-D(u) \# \nabla^{i_{1}} u \# \cdots \# \nabla^{i_{j}} u \nabla^{i_{j+1}} v\right| \\
& \leq\left\{|D(\widetilde{u})-D(u)|\left|\nabla^{i_{1}} \widetilde{u}\right| \cdots\left|\nabla^{i_{j}} \widetilde{u}\right|\right. \\
& \quad+|D(u)|\left|\nabla^{i_{1}}(\widetilde{u}-u)\right|\left|\nabla^{i_{2}} \widetilde{u}\right| \cdots\left|\nabla^{i_{j}} \widetilde{u}\right| \\
& \left.\quad+\cdots+|D(u)|\left|\nabla^{i_{1}} u\right| \cdots\left|\nabla^{i_{j}}(\widetilde{u}-u)\right|\right\}\left|\nabla^{i_{j+1}} v\right| .
\end{aligned}
$$

Studying now the $L^{2}$ norm of this sum, the first term can be bounded as before and it goes to zero as $D(u)$ is continuous from $P^{m}$ to $L^{\infty}$. The $L^{2}$ norm of all the other terms, repeating step by step the previous estimates, using Hölder's inequality and embeddings (4.1)-(4.3), will be estimated by some product

$$
C\|u\|_{P^{m}}^{\alpha}\|\widetilde{u}\|_{P^{m}}^{\beta}\|v\|_{P^{m}}^{\gamma}\|\widetilde{u}-u\|_{P^{m}}^{\sigma} \leq C\|u\|_{P^{m}}^{\alpha}\|\widetilde{u}\|_{P^{m}}^{\beta}\|\widetilde{u}-u\|_{P^{m}}^{\sigma}
$$


for a constant $C$ and some nonnegative exponents $\alpha, \beta, \gamma, \sigma$ satisfying $\alpha+\beta+\gamma+$ $\sigma \leq 1$ and $\sigma>0$. Here we we used the fact that $\|v\|_{P^{m}} \leq 1$.

As $\tilde{u}-u \rightarrow 0$ in $P^{m}$, this last product goes to zero in $L^{2}$, hence uniformly for $\|v\|_{P^{m}} \leq 1$ and inequality (3.12) follows, as claimed. The analysis of the estimates with mixed time/space derivatives is similar and all this argument works analogously for the term $A(u) \cdot \nabla^{2 p} v$.

Then, the Gateaux derivative $d \mathcal{F}_{A}$ is continuous which implies that it coincides with the Frechét derivative, hence $\mathcal{F}_{A} \in C^{1}\left(P^{m} ; P^{m-1}\right)$.

The map $\mathcal{F}_{b}$ can be dealt with in the same way and we are done.

\section{Parabolic Sobolev embeddings}

Proposition 4.1. Let $u \in P^{m}(M, T)$. Then for $r, \ell \in \mathbb{N}$ with $2 p r+\ell \leq 2 m p$, we have

$$
\begin{aligned}
& \left\|\partial_{t}^{r} \nabla^{\ell} u\right\|_{L^{q}(M \times[0, T])} \leq C\|u\|_{P^{m}(M, T)} \text { if } \frac{1}{q}=\frac{1}{2}-\frac{2 p m-\ell-2 p r}{n+2 p}>0 \\
& \left\|\partial_{t}^{r} \nabla^{\ell} u\right\|_{L^{q}(M \times[0, T])} \leq C\|u\|_{P^{m}(M, T)} \text { if } \frac{1}{2}-\frac{2 p m-\ell-2 p r}{n+2 p}=0 \text { and } 1 \leq q<\infty
\end{aligned}
$$

the function $\partial_{t}^{r} \nabla^{\ell} u$ is continuous and

$$
\left\|\partial_{t}^{r} \nabla^{\ell} u\right\|_{C^{0}(M \times[0, T])} \leq C\|u\|_{P^{m}(M, T)} \text { if } \frac{1}{2}-\frac{2 p m-\ell-2 p r}{n+2 p}<0,
$$

where the constant $C$ does not depend on $u$.

Proof. Of course we can write

$$
\begin{aligned}
& P^{m}(M, T) \\
& =L^{2}\left([0, T] ; H^{2 m p}(M)\right) \cap H^{1}\left([0, T] ; H^{2 p(m-1)}(M)\right) \cap \cdots \cap H^{m}\left([0, T] ; L^{2}(M)\right) .
\end{aligned}
$$

By standard interpolation theory, see e.g. [8, Theorem 2.3], we have the continuous immersion

$$
P^{m}(M, T) \hookrightarrow H^{s}\left([0, T] ; H^{2 p(m-s)}(M)\right), \quad \text { for all } s \in[0, m] .
$$

We shall now assume that $\frac{1}{2}-\frac{2 p m-\ell-2 p r}{n+2 p}>0$ and prove inequality (4.1). For $0 \leq \sigma<\frac{1}{2}$ and for any Hilbert space $X$ we have the Sobolev embedding

$$
H^{\sigma}([0, T] ; X) \hookrightarrow L^{q}([0, T] ; X) \quad \text { for } \quad \frac{1}{q}=\frac{1}{2}-\sigma
$$


Then, for $\ell, r \in \mathbb{N}$ with $2 p r+\ell \leq 2 p m$ and for any $s \in\left(m-\frac{\ell}{2 p}-\frac{n}{4 p}, m-\right.$ $\left.\frac{\ell}{2 p}\right] \cap\left[r, r+\frac{1}{2}\right)$, also using the standard Sobolev embeddings on $M$, for every $u \in P^{m}(M, T)$ one gets

$$
\begin{aligned}
\partial_{t}^{r} \nabla^{\ell} u \in H^{s-r}\left([0, T] ; H^{2 p(m-s)-\ell}(M)\right) & \hookrightarrow L^{q}\left([0, T] ; H^{2 p(m-s)-\ell}(M)\right) \\
& \hookrightarrow L^{q}\left([0, T] ; L^{\widetilde{q}}(M)\right),
\end{aligned}
$$

with

$$
\frac{1}{q}=\frac{1}{2}-s+r \quad \text { and } \quad \frac{1}{\widetilde{q}}=\frac{1}{2}-\frac{2 p(m-s)-\ell}{n} .
$$

We now choose $s=\frac{r n+2 p m-\ell}{n+2 p}$ and claim that $s \in\left(m-\frac{\ell}{2 p}-\frac{n}{4 p}, m-\frac{\ell}{2 p}\right] \cap\left[r, r+\frac{1}{2}\right)$. Then

$$
\frac{1}{q}=\frac{1}{\widetilde{q}}=\frac{1}{2}-\frac{2 p m-\ell-2 p r}{n+2 p},
$$

hence for such $q \in \mathbb{R}$ we have

$$
u \in L^{q}\left([0, T] ; L^{q}(M)\right) \simeq L^{q}(M \times[0, T]),
$$

and embedding (4.1) is proven. As for the claim, the inequalities $s \geq r$ and $s \leq$ $m-\frac{\ell}{2 p}$ easily follow from the inequality $2 p r+\ell \leq 2 p m$, while inequality $s<$ $r+\frac{1}{2}$ is equivalent to $\frac{1}{2}-\frac{2 p m-\ell-2 p r}{n+2 p}>0$. This means $\frac{1}{q}>0$ which implies $s>m-\frac{\ell}{2 p}-\frac{n}{4 p}$.

The proof of inequality (4.2) is analogous.

Finally, if $\frac{1}{2}-\frac{2 p m-\ell-2 p r}{n+2 p}<0$, using that for $\sigma>\frac{1}{2}$ one has $H^{\sigma}([0, T] ; X) \hookrightarrow$ $C^{0}([0, T] ; X)$ and that for $\sigma>\frac{n}{2}$ one has $H^{\sigma}(M) \hookrightarrow C^{0}(M)$, for every $u \in$ $P^{m}(M, T)$ we infer

$\partial_{t}^{r} \nabla^{\ell} u \in H^{s-r}\left([0, T] ; H^{2 p(m-s)-\ell}(M)\right) \hookrightarrow C^{0}\left([0, T] ; C^{0}(M)\right) \simeq C^{0}(M \times[0, T])$, for $s=\frac{r n+2 p m-\ell}{n+2 p} \in\left(r+\frac{1}{2}, m-\frac{\ell}{2 p}-\frac{n}{4 p}\right)$. This proves embedding (4.3).

\section{References}

[1] R. AdAms, "Sobolev Spaces", Academic Press, New York, 1975.

[2] S. AGMON, A. DOUGLIS and L. NIRENBERG, Estimates near the boundary for solutions of elliptic partial differential equations satisfying general boundary conditions, Comm. Pure Appl. Math. 12 (1959), 623-727.

[3] T. Aubin, "Some Nonlinear Problems in Riemannian Geometry", Springer-Verlag, 1998.

[4] S. BRENDLE, Convergence of the Yamabe flow for arbitrary initial energy, J. Differential Geom. 69 (2005), 217-278.

[5] M. GIAQUinTA and G. ModicA, Local existence for quasilinear parabolic systems under nonlinear boundary conditions, Ann. Mat. Pura Appl. 149 (1987), 41-59. 
[6] G. Huisken and A. Polden, Geometric Evolution Equations for Hypersurfaces, In: "Calculus of Variations and Geometric Evolution Problems (Cetraro, 1996)", Springer-Verlag, Berlin, 1999, 45-84.

[7] E. KUWERT and R. SCHÄTZLE, Gradient flow for the Willmore functional, Comm. Anal. Geom. 10 (2002), 307-339.

[8] J. L. Lions and E. Magenes, "Non-homogeneous Boundary Value Problems and Applications", Vol. I, Springer-Verlag, New York, 1972.

[9] A. Malchiodi and M. StruWe, $Q$-curvature flow on $\mathbb{S}^{4}$, J. Differential Geom. 73 (2006), $1-44$.

[10] A. Polden, Curves and Surfaces of Least Total Curvature and Fourth-Order Flows, P.h.D. thesis, Mathematisches Institut, Univ. Tübingen, 1996, Arbeitsbereich Analysis Preprint Server - Univ. Tübingen, http://poincare.mathematik.uni-tuebingen.de/mozilla/home.e.html.

[11] H. SCHWETLICK and M. STRUWE, Convergence of the Yamabe flow for "large" energies, J. Reine Angew. Math. 562 (2003), 59-100.

[12] J. J. ShARPLES, Linear and quasilinear parabolic equations in Sobolev space, J. Differential Equations 202 (2004), 111-142.

[13] R. YE, Global existence and convergence of Yamabe flow, J. Differential Geom. 39 (1994), $35-50$.

Scuola Normale Superiore Piazza dei Cavalieri 7

56126 Pisa, Italia

c.mantegazza@sns.it

Centro di Ricerca Matematica

"Ennio De Giorgi"

Scuola Normale Superiore

Piazza dei Cavalieri, 3

56126 Pisa, Italia

luca.martinazzi@sns.it 